\title{
Atuação dos fisioterapeutas no âmbito da Atenção Primária à Saúde (APS) junto a usuários suspeitos ou diagnosticados com COVID-19*: contribuições da Fisioterapia Respiratória
}

Physiotherapy in patients with suspected or confirmed COVID-19 within the context of Primary Health Care $(\mathrm{PHC})^{*}$ : contributions from Respiratory Physiotherapy

DIAS, Claudia Silva'; CAMELIER, Fernanda Warken Rosa ${ }^{2}$; SANTOS, Mara Lisiane de Moraes dos 3 , em nome do Comitê COVID-19 da ASSOBRAFIR.

\begin{abstract}
Resumo
Este documento tem por objetivo apresentar recomendações e informar aos fisioterapeutas e serviços de saúde sobre o papel do fisioterapeuta atuante na Atenção Primária à Saúde (APS) durante a pandemia de COVID-19, com ênfase numa avaliação detalhada e na utilização de técnicas de fisioterapia respiratória com o intuito de ampliar o conhecimento acerca da especialidade nas diferentes realidades da rede de atenção no Brasil. Os fisioterapeutas da APS, ao incorporarem os conhecimentos da fisioterapia respiratória e cardiovascular em seu cotidiano do trabalho, tanto para o planejamento das ações da APS (acolhimento, triagem e encaminhamentos), quanto para o acompanhamento dos usuários dos grupos de risco, dos casos suspeitos ou confirmados da COVID-19, aqueles que cursem com sintomas respiratórios leves, e em condições de pós alta hospitalar, têm papel preponderante no cuidado ampliado e possíveis melhores desfechos clínicos junto aos usuários que estão sob a responsabilidade de suas equipes.
\end{abstract}

Palavras-chave: Fisioterapia; COVID-19; Atenção Primária à Saúde.

\footnotetext{
${ }^{*}$ Revisado por membros do Comitê COVID-19 da ASSOBRAFIR, nomeado por meio do memorando No 003/2020. Esta publicação é uma atualização da Comunicação Oficial "Recursos e técnicas fisioterapêuticas que devem ser utilizadas com cautela ou evitadas em pacientes com COVID-19", chancelada pelo Comitê COVID-19 da ASSOBRAFIR, originalmente escrita pelos mesmos autores e divulgada em 03/06/2020 no endereço eletrônico https://assobrafir.com. br/covid-19-fisioterapia-na-atencao-primaria-a-saude/

${ }^{1}$ Departamento de Fisioterapia, Pontifícia Universidade Católica (PUC) de Minas Gerais, Belo Horizonte, Minas Gerais, Brasil. CSD - https://orcid.org/0000-0002-1205-8862

${ }^{2}$ Departamento de Ciências da Vida (DCV), Universidade do Estado da Bahia (UNEB), Salvador, Bahia, Brasil. FWRC - https://orcid.org/0000-0003-2540-0142

${ }^{3}$ Instituto Integrado de Saúde, Universidade Federal de Mato Grosso do Sul (UFSM), Campo Grande, Mato Grosso do Sul, Brasil. Email: mara.santos@ufms.br MLMS - https://orcid.org/0000-0001-6074-0041
} 


\section{Abstract}

This document aims to present recommendations and inform physiotherapists and health services about the role of physiotherapists within the context of Primary Health Care (PHC) during the COVID-19 pandemic, focusing on a detailed assessment and the use of respiratory physiotherapy techniques in order to increase the knowledge about this speciality in the different realities of the Brazilian healthcare network. PHC physiotherapists, when incorporating the knowledge about respiratory and cardiovascular physiotherapy into their daily work, both for planning PHC actions (reception, screening and referrals) as well as for monitoring patients at risk for poorer health, suspected or confirmed cases of COVID-19, those with mild respiratory symptoms, and those following hospital discharge, have an important role in the expanded care and to contribute to potentially better clinical outcomes in healthcare users who are under the responsibility of the PHC team.

Keywords: Physiotherapy; COVID-19; Primary Health Care.

\section{Contextualização}

O Sistema Único de Saúde (SUS) é o maior sistema de saúde pública de cobertura universal do mundo, e possivelmente o maior patrimônio das políticas públicas do país. No SUS, a Atenção Básica (AB), especificamente por meio das Equipes de Saúde da Família (eSF), ocupa a centralidade das ações para a reorganização do modelo assistencial no país, é a ordenadora das redes de atenção à saúde e incorpora os princípios da Atenção Primária à Saúde (APS). Dados de dezembro de 2019 apontam que a $\mathrm{AB}$ no país era responsável pelo cuidado de 155.875 .540 de pessoas, com cobertura de $74,8 \%$ da população ${ }^{1}$. Nesse cenário, de acordo com dados de março de 2020 do sistema de dados do SUS (DATASUS), há 10.287 fisioterapeutas atuando na APS, seja em eSF ou em equipes do Núcleo Ampliado de Saúde da Família (NASF).

A APS é a principal porta de entrada do SUS e, mediante a elevada cobertura e grande capilaridade desse serviço no país ${ }^{2}$, as equipes que atuam nesses espaços estão em posição estratégica e fundamental no enfrentamento da pandemia de COVID-19. Com a manutenção da longitudinalidade e a coordenação do cuidado nas redes de atenção à saúde, com importante papel na condução dos casos suspeitos e confirmados de COVID-19 com sintomas leves, e também na identificação dos casos mais graves que devem ser manejados em serviços especializados ${ }^{3}$, a APS desempenha importante papel nesse período de pandemia. A COVID-19 é uma doença respiratória infecciosa das vias aéreas superiores e/ou inferiores (pneumonia viral), altamente contagiosa e que pode causar disfunções sistêmicas: respiratória, cardíaca, hepática, renal, neurológica, imunológica, física e psicológica aos usuários $^{4-14}$.

O SARS-CoV-2 é o vírus que causa a infecção, que cursa assintomática ou com sintomas leves em aproximadamente $80 \%$ dos casos, mas pode provocar uma síndrome respiratória aguda capaz de evoluir para insuficiência respiratória grave em 5 a 10\% dos casos. Trata-se de um agravo à saúde recente e por isso ainda há muito a ser esclarecido sobre sua história e evolução natural, bem como sobre o melhor manejo clínico.

Há condições que aumentam o risco para agravamento do estado de saúde frente à COVID-19, como doenças cardíacas (crônicas, isquêmicas, congênita); doenças respiratórias crônicas (doença pulmonar obstrutiva crônica - DPOC, asma mal controlada, doenças pulmonares intersticiais, fibrose cística, displasia broncopulmonar nos lactentes); doenças renais crônicas; usuários em diálise 
(hemodiálise ou diálise peritoneal); imunossupressão por doenças e/ou medicamentos (em vigência de quimioterapia/radioterapia, entre outros medicamentos); transplantados de órgãos sólidos e de medula óssea; diabetes mellitus; pessoas com deficiência por restrições respiratórias, dificuldades nos cuidados pessoais, condições autoimunes, entre outras, e doenças raras ${ }^{15}$; pessoas com doenças cromossômicas e com estados de fragilidade imunológica (ex.: Síndrome de Down), e hábitos de vida como o tabagismo e alcoolismo ${ }^{16}$. A idade avançada, condições crônicas associadas, gestantes e puérperas estão relacionadas à maior letalidade na COVID-19.

As experiências de vários países do mundo têm mostrado que somente com ações extremamente coordenadas entre os cuidados nas comunidades, nos postos de trabalho e nos serviços de saúde que utilizam tecnologias de alta densidade, com os de urgência, emergência e hospitalar, é possível enfrentar de modo efetivo a COVID-19. Nesse contexto, a APS tem papel fundamental no rastreamento, acolhimento, monitoramento, encaminhamento e/ou tratamento das pessoas com suspeita ou diagnóstico da COVID-19 no território adscrito, bem como dos grupos de risco ${ }^{3}$.

Em relação às práticas dos Fisioterapeutas no cuidado dos usuários com COVID-19, foram publicados documentos internacionais norteadores destinados aos profissionais que atuam em ambiente hospitalar e após a alta, a partir da realidade observada na China ${ }^{4,17-19}$. A ASSOBRAFIR também tem contribuído para informação dos fisioterapeutas e divulgou uma série de comunicações oficiais que podem ser encontradas em seu sítio eletrônico*. Entretanto, até o momento, a literatura apresenta poucos documentos que abordam a assistência Fisioterapêutica no âmbito da APS, seja no acolhimento, rastreamento, tratamento ou encaminhamentos para atenção especializada dos usuários com diferentes condições clínicas da COVID-19, dos casos suspeitos e grupos de risco.

É importante salientar que as experiências da China e Itália demonstraram que as pessoas com COVID-19, em fase inicial da doença têm indicação mínima para o uso de técnicas de remoção de secreção brônquica, otimização da ventilação e da oxigenação, o que pode ser diferente para os usuários com fatores de risco e/ou doenças associadas. Esses recursos e estratégias são mais requeridos na assistência fisioterapêutica no ambiente hospitalar (enfermarias e unidades de terapia semi-intensiva e intensiva), quando a frequência de tosse e de secreção pulmonar, demonstradas em um estudo chinês, são, respectivamente, de $67,8 \%$ e $33,7 \%{ }^{20}$. Todos estes recursos, associados ao manejo do suporte ventilatório e mobilização precoce, são fundamentais nas circunstâncias em que o Fisioterapeuta, com as devidas precauções, tem indicação para intervir nos diferentes cenários de tratamento e distintas fases da evolução da doença.

Assim, considerando o papel estratégico da APS para o enfrentamento da pandemia de COVID-19 no Brasil, associado ao expressivo número de profissionais fisioterapeutas generalistas que atuam nesses espaços, a ASSOBRAFIR apresenta orientações para nortear as ações dos fisioterapeutas da APS junto aos usuários e comunidade dos territórios adscritos, e também junto àqueles considerados grupo de risco para desenvolverem complicações a partir da COVID-19. A intenção não é esgotar o assunto; pelo contrário, abrir um canal de debate com os profissionais que atuam na APS sobre as práticas dos fisioterapeutas no manejo e mitigação da infecção e que, muitas vezes, não têm experiência ou formação complementar em fisioterapia respiratória, assim como com especialistas e pesquisadores da área.

\footnotetext{
*https://assobrafir.com.br/covid-19/
} 


\section{As Tecnologias do Cuidado na APS}

A APS constitui um espaço de produção de cuidado que utiliza ferramentas de baixa densidade tecnológica, mas de alta complexidade ${ }^{2}$. É no âmbito da APS que as pessoas têm total autonomia para conduzirem suas vidas e, em situações como a da pandemia da COVID-19, tais peculiaridades tornam-se ainda mais relevantes para que os projetos profiláticos e terapêuticos tenham a adesão dos usuários.

Ainda que a pandemia da COVID-19 seja mundial, os fatores individuais, sociais, culturais, religiosos, entre outros, repercutem em modos singulares de viver a doença ou o risco de adoecer. Os diferentes contextos interferem nas distintas maneiras de encarar e viver esse momento, e é no cuidado no território - no âmbito da APS - que tais particularidades são cruciais para o sucesso no enfrentamento da pandemia. Nessa perspectiva, destacamos que as tecnologias leves e as leveduras devem estar na centralidade da relação entre o profissional e o usuário. As tecnologias leves são aquelas utilizadas para favorecer o cuidado: escuta, empatia, porosidade, reconhecimento dos distintos saberes, das diferentes realidades, dos conhecimentos prévios trazidos por cada usuário. As tecnologias leve-duras são relativas aos conhecimentos necessários para a atuação terapêutica junto aos usuários, famílias e comunidade ${ }^{21}$.

As equipes de saúde na APS têm o desafio de promover o cuidado de modo que as singularidades dos usuários sejam consideradas, com atos cuidadores capazes de ampliar as potências de vida e favorecer o combate à pandemia com o enfrentamento de problemas que não podem ser resolvidos. Nesse contexto, a vulnerabilidade social ocupa lugar de destaque, e a extrema objetivação e generalização de ações sobre o corpo biológico, com prescrições higienizadoras e de isolamento no domicílio, podem deixar passar outros elementos que são constitutivos da produção da vida e que não são incluídos, trabalhados, tanto na tentativa de compreender a situação, como nas intervenções para enfrentá-la ${ }^{22}$.

Assim, não podemos deixar de propor a seguinte reflexão: as práticas dos Fisioterapeutas e das equipes na APS devem operar a partir das melhores evidências para o enfrentamento da pandemia da COVID-19, associadas a práticas que dialoguem com a diversidade das realidades em que as pessoas vivem e das diferentes formas de viver. Trazer para a cena do cuidado as tecnologias leves ao mesmo tempo em que é desafiador, é essencial para a adesão dos usuários e potencialização dos projetos terapêuticos para o enfrentamento da pandemia da COVID-19.

\section{Acolhimento, rastreamento e ações}

O Fisioterapeuta, como membro da equipe da APS, deverá incorporar em seu processo de trabalho as recomendações do Ministério da Saúde, disponibilizadas no "Protocolo de manejo clínico do Coronavírus (Covid-19) na APS" ${ }^{3}$ que atendam as melhores evidências científicas disponíveis e as orientações da Organização Mundial da Saúde (OMS). Nesse cenário, para um cuidado efetivo é essencial acompanhar as atualizações disponibilizadas por essas instituições. Cada município tem autonomia para organizar os próprios fluxos e intervenções nos serviços de saúde, considerando as diretrizes acima mencionadas, as particularidades locais da organização da rede de atenção à saúde e as características epidemiológicas da população.

É importante que o Fisioterapeuta participe e contribua com o planejamento e com as ações a serem desenvolvidas no território adscrito, o qual constitui-se a partir de uma delimitação espacial, 
onde vive uma população com um perfil epidemiológico, social, cultural e demográfico. Tais características tornam o território dinâmico, sempre em movimento, no qual a população adota estratégias próprias para o enfrentamento da pandemia de COVID-19. A partir da realidade local, a equipe da APS deverá ponderar sobre como ajudar as famílias a seguirem as recomendações de isolamento social e comportamentais para enfrentamento da pandemia. Nesse contexto, vale ressaltar a importância das abordagens que intensifiquem a busca ativa de sintomáticos respiratórios, com vigilância e monitoramento de regiões mais aglomeradas e vulneráveis

Até o momento, o comprometimento do sistema respiratório é o mais preocupante na COVID-19 e, assim, exige uma abordagem específica e resolutiva o mais precoce possível, direcionada aos usuários dos grupos de risco, com síndrome gripal ou com o diagnóstico de COVID-19 (Quadro 1) em tratamento no domicílio.

Quadro 1 | Definições de Síndrome Gripal, Síndrome Respiratória Aguda Grave e diagnóstico laboratorial da COVID-193.

SÍNDROME GRIPAL (SG): indivíduo com quadro respiratório agudo, caracterizado por sensação febril ou febre*, mesmo que relatada, acompanhada de tosse OU dor de garganta OU coriza OU dificuldade respiratória. *Na suspeita de Covid-19, a febre pode não estar presente.

- EM CRIANÇAS: considera-se também obstrução nasal, na ausência de outro diagnóstico específico.

- EM IDOSOS: a febre pode estar ausente. Deve-se considerar também critérios específicos de agravamento como sincope, confusão mental, sonolência excessiva, irritabilidade e inapetência.

SÍNDROME RESPIRATÓRIA AGUDA GRAVE (SRAG): Síndrome Gripal que apresente dispneia/desconforto respiratório OU Pressão persistente no tórax OU saturação de $\mathrm{O}_{2}$ menor que $95 \%$ em ar ambiente OU coloração azulada dos lábios ou rosto.

- EM CRIANÇAS: além dos itens anteriores, observar os batimentos de asa de nariz, cianose, tiragem intercostal, desidratação e inapetência.

CRITÉRIO LABORATORIAL - caso suspeito de SG ou SRAG com teste de:

- Biologia molecular (RT-PCR em tempo real, detecção do vírus SARSCoV2): - Doença pelo Coronavírus 2019: com resultado detectável para SARS-CoV2. Amostra clínica coletada, preferencialmente até o sétimo dia de início de sintomas.

- Imunológico (teste rápido ou sorologia clássica para detecção de anticorpos para o SARS-CoV2): com resultado positivo para anticorpos IgM e/ou lgG. Em amostra coletada após o sétimo dia de início dos sintomas.

CRITÉRIO CLÍNICO-EPIDEMIOLÓGICO: caso suspeito de SG ou SRAG com histórico de contato próximo ou domiciliar, nos últimos 7 dias antes do aparecimento dos sintomas, com caso confirmado laboratorialmente para COVID19 e para o qual não foi possível realizar a investigação laboratorial específica. 
Nesse cenário, o Fisioterapeuta da APS deverá incorporar em seu cotidiano práticas da fisioterapia respiratória, adotando as medidas de prevenção da transmissão do coronavírus e de tratamento dos casos confirmados e suspeitos que cursam com sintomas leves ${ }^{3}$, orientadas pela Organização Mundial da Saúde e pelas melhores evidências disponíveis. Deverá ser capaz de avaliar sinais e sintomas quando diante de casos suspeitos ou confirmados de COVID-19, como: tempo de diagnóstico ou presença de sintomas, temperatura corporal $(>37,8 \mathrm{oC})$, presença e característica da tosse, presença e intensidade da dispneia, tempo desde o início da dispneia, saturação de pulso de oxigênio ( $\leq 94 \%)$, pressão arterial $(<90 / 60 \mathrm{mmHg}$ ou $>140 / 90 \mathrm{mmHg})$, presença de mialgia e fadiga ${ }^{3}$. Publicações recentes também associam a COVID-19 a sintomas como anosmia (distúrbios do ouvido, nariz e garganta), vômito, diarreia, entre outros ${ }^{23,24}$.

A avaliação fisioterapêutica, que envolve a anamnese e o exame físico, com todos os cuidados em relação a proteção individual, é um recurso imprescindível para obtenção de informações e dados que norteiam a assistência fisioterapêutica nos diferentes níveis de atenção à saúde. No contexto da pandemia de COVID-19, e de acordo com os fluxos e direcionamentos de documentos oficiais e das melhores evidências científicas disponíveis, busca-se aqui enfatizar aspectos relevantes da avaliação fisioterapêutica no âmbito da APS.

A dispneia refere-se à percepção subjetiva do aumento do trabalho respiratório ${ }^{25}$. Com o objetivo de qualificar e quantificar este sintoma, assim como acompanhar a evolução da piora da dispneia, sugere-se a padronização da avaliação utilizando a Escala de Dispnéia do Medical Research Council (MRC) ${ }^{26}$ (Quadro 2).

Quadro 2 | Escala modificada de dispneia do Medical Research Council (mMRC).

\section{Você tem falta de ar?}

\section{Grau 0 (esperada)}

Grau 1 (leve)

Grau 2 (moderada)

Grau 3 (acentuada)

Grau 4 (muito acentuada) com atividades extraordinárias, tais como correr, carregar cargas pesadas no plano ou cargas leves subindo escadas. com atividades maiores, tais como subir ladeira muito inclinada, 2 ou mais andares ou carregando pacote pesado de compras no plano. com atividades moderadas, tais como: subir 1 andar, caminhar depressa no plano, ou carregar cargas leves no plano. com atividades leves, tais como: tomar banho, andar uma quadra em passo regular.

em repouso ou para se vestir ou caminhar poucos passos devagar.

Outro sintoma a ser observado é a tosse, que é um importante reflexo de proteção das vias áreas. No momento da avaliação, deve-se obter informações acerca da sua efetividade e presença de expectoração25. É relevante o fisioterapeuta orientar aos membros da equipe de saúde a não perguntar apenas se há presença de tosse ao paciente sintomático gripal ou caso confirmado de COVID-19, mas contextualizar a presença deste sintoma previamente a ida ao serviço de saúde.

São exemplos para esta abordagem: 


\begin{tabular}{|l|l|}
\hline Você habitualmente tosse ou pigarreia? & Se a resposta for afirmativa, prosseguir \\
\hline $\begin{array}{l}\text { Sua tosse está pior em relação ao que } \\
\text { normalmente apresenta? }\end{array}$ & Posteriormente \\
\hline Você elimina catarro? & \\
\hline
\end{tabular}

O exame físico deve ser realizado de maneira criteriosa, e precisam ser avaliados, além dos sinais e sintomas, os seguintes parâmetros:

- Frequência respiratória (FR): deve ser avaliada com o usuário em repouso, em decúbito dorsal ou sentado, por pelo menos 30 segundos ${ }^{25}$. A presença de taquipneia $(\mathrm{FR}>30$ respirações por minuto no adulto) está relacionada a gravidade dos sintomas para a Síndrome Gripal ${ }^{3}$.

- Sinais de desconforto respiratório: esta avaliação deve ser realizada, preferencialmente, no usuário com o tórax desnudo. O desconforto respiratório é caracterizado pelos músculos respiratórios que estão executando sua função em excesso ${ }^{27}$, com sobrecarga. Avaliar a presença de tiragens, que são movimentos de retração da pele/músculo que recobrem a parede torácica durante o movimento inspiratório (isso ocorre devido à queda pressórica abrupta gerada pela musculatura respiratória). As tiragens podem ser de intercostais, supraclaviculares ou subcostais, dependendo da sua localização $0^{28}$. A presença deste achado está associada à fadiga muscular respiratória, e indica gravidade da Síndrome Gripal ${ }^{3}$.

- Ausculta pulmonar: geralmente é realizada com o usuário em posição sentada, com o tórax exposto, orientando, dentro do possível, que ele realize respiração oral. Com o estetoscópio todo o tórax deve ser percorrido, sendo realizando a comparação dos sons respiratórios entre os hemitórax ${ }^{25}$. O Fisioterapeuta deve estar atento na avaliação para identificar a presença ou ausência de sons normais e/ou não, como crepitações, sibilos e ron $\cos ^{29}$. A presença de roncos, de acordo com o "Protocolo de Manejo Clínico do Coronavírus (COVID-19) na Atenção Primária à Saúde"

- Oximetria de pulso $\left(\mathrm{SpO}_{2}\right)$ : avaliação não invasiva por meio do oxímetro de pulso ${ }^{30}$. A $\mathrm{SpO}_{2}<95 \%$ em ar ambiente está associada com a gravidade da Síndrome Gripal ${ }^{3}$. Caso o paciente não tenha história de doenças crônicas, como as pneumopatias, cardiopatias, condições metabólicas ou deficiência física e a saturação for inferior a $88 \%$, este deverá ser encaminhado imediatamente para uma unidade de internação hospitalar para tratamento do quadro.

- Avaliar se o usuário faz uso prótese ventilatória e/ou suplementação de oxigênio mesmo que em ventilação espontânea.

Os usuários com suspeita de COVID 19 (porém assintomáticos) ou com síndrome gripal, deverão ser contatados pelo menos uma vez a cada 48 horas, por telefone e se necessário, presencialmente, para que seja investigado quanto aos critérios clínicos e qualquer alteração da condição clínica. E os usuários com diagnóstico confirmado deverão ser monitorados pela equipe de profissionais de saúde, por telefone, todos os dias ${ }^{3}$. 


\section{Educação em Saúde}

A prática de educação em saúde deverá ser uma rotina dos profissionais de saúde e propiciará que o usuário aprenda sobre a doença, suas repercussões, estratégicas de prevenção e o processo de tratamento por meio de orientações utilizando-se metodologias diversas, como conversas com os usuários que estejam presentes nas Unidades de Saúde, folders, vídeos, áudios, podcasts, lives, chamadas telefônicas por vídeo, mensagens em redes sociais da unidade de saúde, manuais e cartilhas divulgados por meios de comunicação formais de órgãos de saúde em nível nacional, estadual e/ou municipal, entidades de classe e associações profissionais, dentre outros. Para ampliar o impacto dessas ações é importante que a linguagem seja acessível aos usuários e que os canais de informação confiáveis estejam ao alcance dos usuários e seus familiares.

Consideramos importante reforçar algumas orientações/recomendações aos usuários que podem ser utilizadas pelos Fisioterapeutas e toda a equipe da APS.

\section{Orientações sobre medidas de responsabilidade sanitária}

1. O isolamento social é a forma mais adequada de evitar o contágio e todas as vezes que o usuário entrar em contato com outras pessoas deverá usar a máscara cirúrgica, de tecido ou tecido-não-tecido $(\mathrm{TNT})^{31}$.

2. A máscara cirúrgica deverá ser substituída a cada 2-3 horas ou quando estiver úmida, e deve ser descartada em saco plástico fechado e condicionada em lixeira.

3. As máscaras "caseiras" podem ser utilizadas em ambientes onde o distanciamento for inviável, desde que confeccionadas, utilizadas e higienizadas de maneira correta. Segundo a OMS, devem ter pelo menos três camadas: a camada exterior deve ser feita de um material resistente à água, como o polipropileno, poliéster ou uma mistura deles; a camada do meio deve agir como um filtro e pode ser feita de um material sintético, como o polipropileno, ou de uma camada extra de algodão; a camada interior deve ser feita de um material que absorva a água, como o algodão. O importante é que a máscara seja feita nas medidas corretas cobrindo totalmente a boca e nariz e que estejam bem ajustadas ao rosto, sem deixar espaços entre o rosto e a máscara ${ }^{32}$. Trocar quando a máscara estiver úmida. Deve ser lavada pelo próprio indivíduo com sabão e/ou água sanitária, deixando de molho por cerca de 30 minutos $^{31}$.

4. Seguem as recomendações da OMS para o uso das máscaras: lavar as mãos com água e sabão ou use álcool em gel antes e após a utilização da máscara, a qual deverá ser examinada previamente ao uso, e se estiver danificada deverá ser descartada; ao retirar a máscara, incline-se ligeiramente para a frente e pegue na máscara pelos elásticos, na parte que está atrás da orelha, sem tocar a frente. Não toque na máscara enquanto estiver usando, para evitar contaminação, e se tocar na máscara acidentalmente, limpe as mãos ${ }^{32}$.

5. Os usuários com doenças crônicas, como as pneumopatias, cardiopatias, condições metabólicas ou deficiência física deverão manter a medicação de controle prescrita pelo médico e qualquer dúvida o mesmo ou um familiar deverá contatar, por telefone, o médico de referência ou a unidade de referência.

6. Etiqueta da tosse: ao tossir ou espirrar, deve-se cobrir o nariz e a boca com a parte interna do cotovelo flexionado ou com um lenço, preferencialmente descartável; caso não tenha um lenço, no momento, tossir ou espirrar na parte de cima da manga da blusa/camisa; em caso de tosse produtiva, orientar a eliminação da secreção em um lenço, que deverá ser 
descartado em sacos de plástico, fechados e colocados em uma lixeira. É preciso higienizar as mãos em seguida com água e sabão ou álcool gel ${ }^{3}$.

No Quadro 3 estão descritas algumas sugestões sobre exercícios e cuidados que podem ser orientados aos usuários com suspeita ou confirmação de COVID-19 sintomáticos ou com síndrome gripal ou usuários dos grupos de risco.

Quadro 3 | Exercícios e cuidados que podem ser orientados aos usuários com suspeita ou confirmação de COVID-19 sintomáticos ou com síndrome gripal ou usuários dos grupos de risco.

\begin{tabular}{|c|c|c|c|}
\hline & Justificativa & Sugestões & Cuidados \\
\hline $\begin{array}{l}\text { Estável sem } \\
\text { sinais e } \\
\text { sintomas }\end{array}$ & $\begin{array}{l}\text { Devido ao início repentino do período de } \\
\text { quarentena/isolamento social os usuários } \\
\text { poderão desenvolver: } \\
\text { - mudança para um estilo de vida menos } \\
\text { ativo; } \\
\text { - redução da força muscular; } \\
\text { - risco aumentado de trombose venosa } \\
\text { profunda; } \\
\text { - alteração do estado emocional com } \\
\text { risco de falta de colaboração ou o } \\
\text { abandono do tratamento associado ao } \\
\text { medo da doença. } \\
\\
\text { O estado fisicamente ativo influencia } \\
\text { positivamente na saúde física e mental, e } \\
\text { melhora componentes da aptidão física } \\
\text { (cardiorrespiratória, força, agilidade de } \\
\text { coordenação). }\end{array}$ & 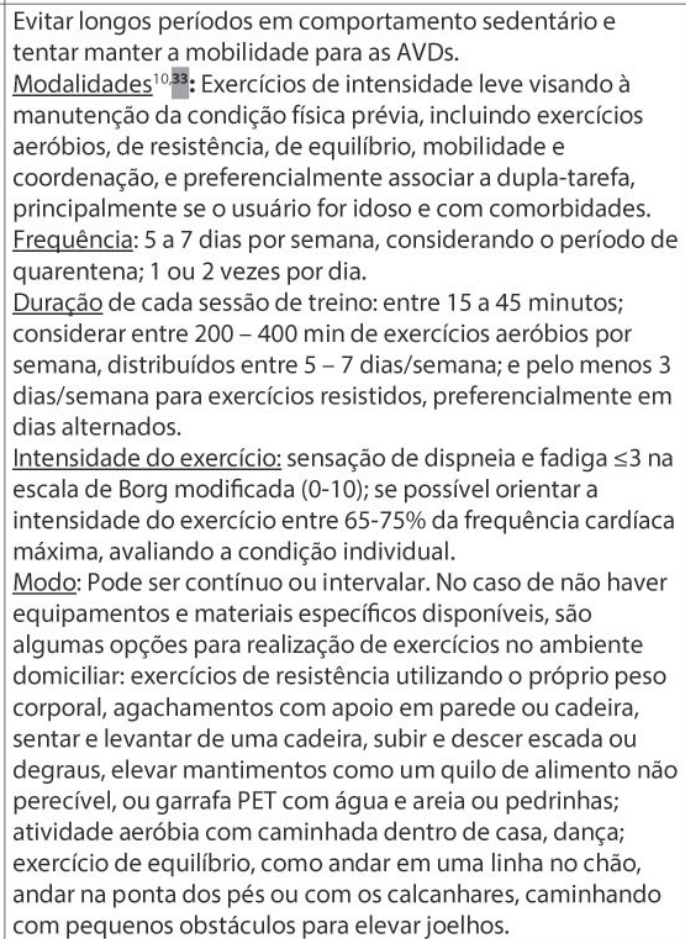 & $\begin{array}{l}\text { - O usuário deverá estar } \\
\text { sem febre, sem fadiga e } \\
\text { dores musculares e sem } \\
\text { dispneia importante. } \\
\text { - Manutenção das horas } \\
\text { de sono, dieta e } \\
\text { hidratação adequadas e } \\
\text { interrupção do hábito de } \\
\text { fumar devem ser } \\
\text { priorizados. } \\
\text { - É aconselhável que no } \\
\text { dia após o exercício físico } \\
\text { o usuário não sinta } \\
\text { fadiga adicional. } \\
\text { - Se idoso ou com déficit } \\
\text { de equilíbrio, atenção em } \\
\text { relação às quedas. }\end{array}$ \\
\hline $\begin{array}{l}\text { Aumento da } \\
\text { dispneia }\end{array}$ & $\begin{array}{l}\text { - Usuário sem doença prévia: } \\
\text { Relata fadiga e/ou falta de ar para AVD. } \\
\text { - Usuário com doença prévia: } \\
\text { Relata piora da fadiga/ou da falta de ar. }\end{array}$ & $\begin{array}{l}\text { Exercícios respiratórios para controle e consciência da } \\
\text { respiração: } \\
\text { Técnica: respiração diafragmática. Sugerimos orientar o } \\
\text { usuário inspirar lentamente pelo nariz e tentar elevar o } \\
\text { abdome, e expirar lentamente pela boca com os lábios } \\
\text { franzidos ou entre os dentes. } \\
\text { Técnicas de conservação de energia: Sugerimos orientar o } \\
\text { usuário a planejar as atividades do dia a dia dando prioridade } \\
\text { para as que forem mais importantes, intercale com períodos } \\
\text { de repouso e caso seja necessário faça os exercícios } \\
\text { respiratórios entre as atividades; tarefas que demandam mais } \\
\text { energias faça quando estiver se sentindo melhor. }\end{array}$ & $\begin{array}{l}\text { Realizar quando estiver } \\
\text { sozinho, devido ao risco } \\
\text { de disseminação do } \\
\text { vírus, em um ambiente } \\
\text { bem ventilado. } \\
\text { Realizar com a utilização } \\
\text { de máscara cirúrgica, se } \\
\text { estiver com o } \\
\text { profissional, e com } \\
\text { distanciamento mínimo } \\
\text { de } 2 \mathrm{~m} \text {. }\end{array}$ \\
\hline $\begin{array}{l}\text { Aumento da } \\
\text { tosse }\end{array}$ & $\begin{array}{l}\text { - Devido à redução da mobilidade pode } \\
\text { haver retenção de secreção, } \\
\text { principalmente em usuários que tenham } \\
\text { histórico de acúmulo de secreção por } \\
\text { doença prévia. } \\
\text { - A maioria dos usuários com COVID-19 } \\
\text { apresenta como principal problema } \\
\text { hipoxemia e tem essencialmente tosse } \\
\text { seca, que pode ocorrer em episódios } \\
\text { sucessivos, levando o usuário a sensação } \\
\text { de fadiga. }\end{array}$ & $\begin{array}{l}\text { As técnicas com objetivo de remoção de secreção brônquica } \\
\text { não são indicadas em usuários que apresentem tosse seca, o } \\
\text { que ocorre na maioria dos casos da COVID-19. } \\
\text { Eventualmente, os usuários podem apresentar tosse } \\
\text { produtiva quando houver alguma doença respiratória prévia } \\
\text { associada, nos casos de tabagismo ou em algumas situações } \\
\text { menos frequentes da COVID-19 ou Síndrome Gripal. Nesse } \\
\text { cenário, podem ser indicadas com cautela técnicas de } \\
\text { remoção de secreção brônquica. A escolha da melhor técnica } \\
\text { deve ser avaliada pelo fisioterapeuta e individualizada, } \\
\text { considerando a especificidade de cada usuário e de suas } \\
\text { condiçōes clínicas no momento da intervenção. }\end{array}$ & $\begin{array}{l}\text { - Utilizar etiqueta da } \\
\text { tosse. } \\
\text { - Utilizar proteção } \\
\text { individual. } \\
\text { - Realizar em ambiente } \\
\text { bem ventilado. } \\
\text {-Mudança do aspecto da } \\
\text { secreça requer procura } \\
\text { médica. }\end{array}$ \\
\hline $\begin{array}{l}\text { O que } \\
\text { deve-se evitar }\end{array}$ & \multicolumn{3}{|c|}{$\begin{array}{l}\text { Evitar o uso de inaladores domésticos para fins de umidificação e fluidificação de secreções. Sugere-se o uso de inaladores } \\
\text { dosimetrados e espaçadores para a administração de broncodilatadores, quando necessário, seguindo recomendações médicas. }\end{array}$} \\
\hline
\end{tabular}


O apoio matricial, quando necessário, deve ser utilizado como estratégia para ampliar e qualificar a atuação dos Fisioterapeutas e profissionais da equipe de APS no cuidado respiratório aos usuários do território, levando para esse cenário de atenção à saúde conhecimentos inerentes à especialidades profissionais de fisioterapia respiratória e fisioterapia cardiovascular, os quais serão úteis no contexto da pandemia da COVID-19. Nessa perspectiva, sugerimos parcerias entre Fisioterapeutas da APS e Fisioterapeutas especialistas em fisioterapia respiratória e fisioterapia cardiovascular que trabalham como docentes em cursos de Fisioterapia de Universidades situadas nas regiões onde as equipes atuam, assim como nas Unidades Regionais, Núcleos e Grupos de Estudo da ASSOBRAFIR, os quais podem ser solicitados como consultores especialistas junto às equipes da APS.

Na mesma direção, os Fisioterapeutas podem utilizar o matriciamento junto aos Agentes Comunitários de Saúde (ACS) e demais profissionais da equipe de APS, com orientações relativas à identificação de sinais e sintomas que possam corresponder ao aparecimento de sintomas ou agravamento do quadro respiratório de usuários do território, identificados durante as visitas domiciliares ou via relatos durante os contatos por telefone, e também podem reforçar as orientações sobre as melhores práticas descritas neste documento.

Outra frente de trabalho que será desafiante aos profissionais da APS é o acolhimento e assistência aos usuários após hospitalização decorrente da COVID-19. O recente relatório elaborado por Fisioterapeutas envolvidos no Comitê da Sociedade Respiratória Européia (ERS) recomenda assistência multiprofissional no âmbito da comunidade aos usuários que foram hospitalizados por COVID-19, sempre que necessário ${ }^{34}$. A OMS recomenda a mobilização ativa do paciente crítico de COVID-19, quando é seguro fazê-lo, desde a fase inicial da doença até após o quadro agudo, iniciando no ambiente hospitalar e progredindo para o domicilio ou centro de reabilitação para dar continuidade ao processo de reabilitação com objetivo de diminuir sintomas e incapacidades, e ganhar funcionalidade ${ }^{35}$. A oferta de programas de reabilitação nas comunidades locais favorece o cuidado a longo prazo $^{36}$ e, nessa perspectiva, as equipes da APS ocupam lugar privilegiado, ao operarem na lógica do cuidado no território, centrada no usuário e por constituírem uma rede de serviços de saúde altamente capilarizada no país.

A reabilitação do paciente que teve COVID-19 deverá ser uma intervenção abrangente com base em avaliação completa do usuário, seguida de intervenções personalizadas que incluem exercícios de treinamento e educação e mudança de comportamento, projetadas para melhorar a condição física e psicológica dos usuários e promover a adesão a longo prazo aos comportamentos de melhoria da saúde. Diante das evidências descritas previamente, em condições de saúde similares, a reabilitação abrangente parece ser uma estratégia promissora a esta parcela de usuários.

Caso a reabilitação seja realizada na APS, e não na atenção secundária ou terciária, os itens a seguir se fazem necessários:

- Avaliação individual (dispneia, fadiga, necessidade de oxigênio, disfunções cardiovasculares, outras doenças prévias etc.);

- Avaliação da função pulmonar, capacidade de exercício e funcional, função muscular, equilíbrio e qualidade de vida relacionada à saúde deve ser realizada somente quando possível;

- Avaliação ambiental (possibilidade de mobilidade segura);

- Avaliação nutricional, psicológica e social (quando necessária); 
- Elaborar propostas de reabilitação direcionadas aos usuários pós COVID-19 que apresentem disfunção ventilatória e/ou descondicionamento físico persistente, como programas domiciliares com exercícios progressivos, orientações sobre mudanças de comportamento e adequação do ambiente, reconhecimento de sinais de alerta em relação à piora do quadro clínico36 e/ou desenvolvimento de complicações, e técnicas de conservação de energia.

Sugestões de programa de intervenção (deverá ser individualizado e dependente das condições clínicas e funcionais do usuário):

- Inicialmente serão incluídos exercícios físicos de baixa intensidade (incluindo fortalecimento funcional) progredindo de acordo com a melhora da capacidade de realização dos exercícios e ganho funcional, conforme descrito no Quadro 3.

Cuidados e recomendações:

- A equipe deverá evitar visitas domiciliares a usuários infectados ou suspeitos para avaliação e assistência, sendo recomendados acompanhamento por contato telefônico (incluir a possibilidade de vídeo chamada) e disponibilização de materiais educativos como cartilhas, folders, vídeos, podcasts e áudios de orientações gerais e/ou individualizadas, dentre outros recursos disponíveis.

- Caso seja necessário a visita domiciliar, esta deverá ser realizada com total segurança (utilização de EPIs) para a equipe e para o usuário e seus familiares, conforme orientações publicadas .

- O encaminhamento de usuários que tiveram diagnóstico de COVID-19 ou suspeitos para serem assistidos nos centros de reabilitação só deverá ser realizado após cura documentada da doença.

- As equipes NASF poderão operar como equipes multidisciplinares itinerantes com programas de reabilitação para os usuários após Covid-19, em ambientes comunitários dos distintos territórios sob sua responsabilidade.

- As equipes deverão apoiar a transição entre hospital e domicílio dos casos mais complexos, que exijam um acompanhamento diferenciado, como por exemplo pessoas com problemas de saúde preexistentes, doenças crônicas, deficiência, idosos, que estejam em oxigenioterapia, entre outros ${ }^{36}$.

- Estabelecer parcerias com os equipamentos sociais do território36, utilizando infraestrutura, recursos materiais e profissionais que contribuam para a ampliação do acesso à reabilitação aos usuários acometidos pela Covid-19.

\section{Considerações Finais}

As experiências do enfrentamento da pandemia com o cuidado nos territórios, no âmbito da APS ainda são pouco divulgadas e compartilhadas, com evidências limitadas. No campo da fisioterapia a realidade é a mesma. Entretanto, acredita-se na potência do cuidado nesse ponto da rede de atenção à saúde no Brasil, que é a principal porta de entrada dos usuários no sistema de saúde. Tal abordagem exige a atuação de uma equipe multiprofissional, e participação ativa das comunidades e grupos sociais. 
Os Fisioterapeutas da APS, ao incorporarem em seu cotidiano do trabalho os conhecimentos da fisioterapia respiratória e cardiovascular, tanto para o planejamento das ações da APS (acolhimento, triagem e encaminhamentos), como para o acompanhamento dos usuários dos grupos de risco, dos casos suspeitos ou confirmados da COVID-19 e que cursem com sintomas respiratórios leves, têm papel preponderante no cuidado ampliado e possíveis melhores desfechos clínicos junto aos usuários que estão sob a responsabilidade de suas equipes.

Após a alta hospitalar, a intervenção fisioterápica em domicílio e na comunidade, no contexto da reabilitação, têm se apresentado como uma necessidade importante dos usuários. No futuro, a disponibilidade de novos conhecimentos sobre o impacto da doença e suas repercussões respiratórias, cardiovasculares, metabólicas e sistêmicas irão nortear com maior precisão a tomada de decisões sobre as intervenções da fisioterapia pós COVID-19, o processo de reabilitação e a reinserção desses na comunidade.

Finalmente, cabe ressaltar que a atuação das equipes da APS nos territórios é extremamente ampla e complexa, e que esse documento pretende abrir o debate sobre a contribuição da Fisioterapia Respiratória e Cardiovascular ao processo de trabalho dos Fisioterapeutas da APS. 
Figura 1 | Fluxo acerca do papel do profissional Fisioterapeuta no contexto da Atenção Primária à Saúde (APS).

\section{COVID-19: Papel o Fisioterapeuta na Atenção Primária à Saúde (APS)}

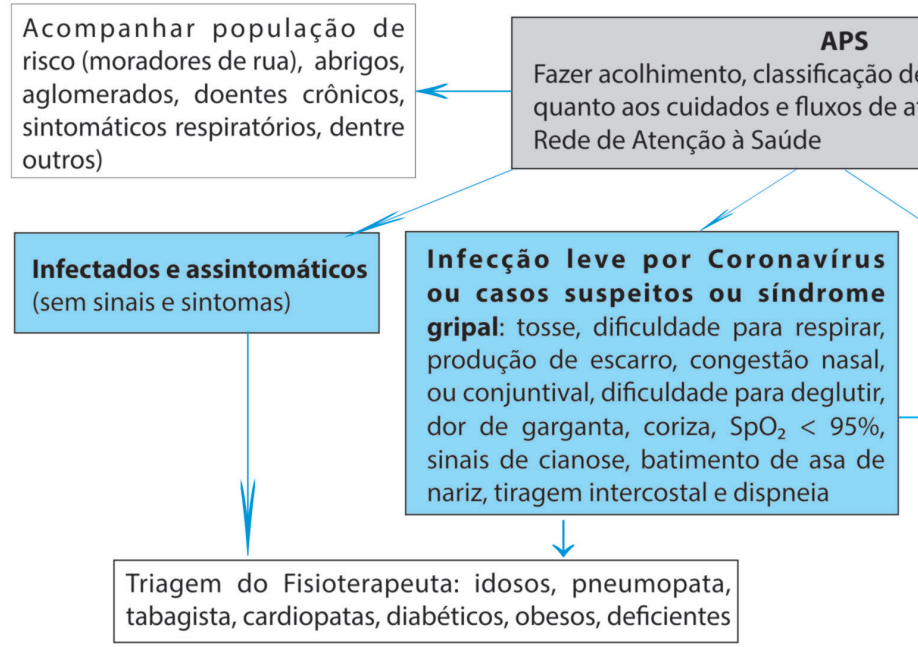

Acompanhar população de risco (moradores de rua), abrigos, aglomerados, doentes crônicos, sintomáticos respiratórios, dentre outros) tabagista, cardiopatas, diabéticos, obesos, deficientes
Autoria:

Claudia Silva Dias

Fernanda Warken Rosa Camelier Mara Lisiane de Moraes dos Santos

Orientar forma de transporte, pronto atendimento, hospital - de acordo com o fluxo regional

Infecção grave/muito grave

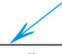

Incertezas quanto a evolução do quadro respiratório pós hospitalização por COVID-19

Acompanhamento domiciliar/Reabilitação Pulmonar Capacitação da equipe

Elaboração de Projetos Terapêuticos - matriciamento

QUADRO 1
1 Notificação dos casos suspeitos e
confirmados (plataforma e-SUS VE
e prontuário
2 Orientações aos usuários quanto
a cuidados específicos:
- Isolamento social e familiar
- Uso de máscaras quando em
contato com familiares
- Manter distanciamento de 2
metros das pessoas
- Higiene pessoal e domiciliar
- Evitar tocar nas mucosas
- Desinfetar objetos
- Hidratação/alimentação
adequada/tempo de sono
adequado/exposição ao sol
- Exercícios respiratórios
- Exercícios gerais leves e
caminhada no domicílio
- Vacinação
- Cuidados com a saúde emocional
3 Monitoramento a cada 48h - via
telefone/ACS

Exercícios Respiratórios

\section{QUADRO 2}

1 Orientações aos usuários quanto a cuidados específicos (conforme descrito QUADRO 1)

2 Avaliação da condição respiratória

- Sinais e sintomas (dispneia, traquipnéia, cianose

e esforço respiratório

- Auscuta respiratória e exames complementares: radiografia, tomografia computadorizada e oximetria

- Avaliar níveis pressóricos e condição mental 3 Orientações para os cuidados ventilatórios

- Exercício respiratório

- Exercícios de MMSS e MMII se estável

- Na presença de secreção - etiqueta de tosse

- Priorizando a Humanização do cuidado

4 Avaliar condição do domicílio (ventilação, aglomeração, mofo, baratas, presença de área externa

5 Monitorização diária - via telefone ou ACS (deverão visitar usuários vulneráveis): investigar sinais e sintomas respiratórios e piora dos sintomas em pneumopatas (hipersecreção, mudança da coloração da secreção, piora da dispneia) 6 Explicar sobre o fluxo de assistência (caso haja mudança do quadro)
Nas unidades o Fisioterapeuta poderá:

- Realizar orientações em sala de espera quanto a prevenção da COVID-19

- Utilizar a TELESAÚDE

- Assegurar a continuidade da assistência frente as demandas das unidades

OBS: As atividades deverão ser realizadas sem aglomeração e com os profissionais devidamente paramentados e os ambientes higienizados

\section{ORIENTAÇÕES QUANTO AOS CUIDADOS VENTILATÓRIOS}




\section{Referências}

1. Brasil. Ministério da Saúde. EgESTOR: Relatório Histórico de Coberta - equipes de saúde da família (eSF) e de equipes de Atenção Básica (eAB) EgESTOR [Internet]. Brasília: Ministério da Saúde; 2020 [cited 2020 Mar 05]. Available from: https://egestorab.saude.gov.br/paginas/acessoPublico/relatorios/ relHistoricoCoberturaAB.xhtml

2. Brasil. Ministério da Saúde. Atenção Básica [Internet]. Brasília: Ministéro da Saúde; 2017 [cited 2020 Mar 05] Available from: https://www.saude.gov.br/artigos/770-sistema-nacional-de-saude/40315-atencao-basica

3. Brasil. Ministério da Saúde. Protocolo do Manejo Clínico do Coronavírus (COVID-19) na Atenção Primária à Saúde (APS) [Internet]. Brasília: Ministério da Saúde; 2020 [cited 2020 Mai 15]. Available from: http://189.28.128.100/dab/docs/portaldab/documentos/20200504 ProtocoloManejo ver09.pdf

4. Chinese Association of Rehabilitation Medicine; Respiratory Rehabilitation Committee of Chinese Association of Rehabilitation Medicine; Cardiopulmonary Rehabilitation Group of Chinese Society of Physical Medicine and Rehabilitation. Recommendations for respiratory rehabilitation of COVID-19 in adult. Zhonghua Jie He He Hu Xi ZaZhi (Chinese Journal of Tuberculosis and Respiratory Diseases) 2020; 43(4):308-314 (abstract). doi: 10.3760/cma.j.cn112147-20200228-00206.

5. Bansal M. Cardiovascular disease and COVID-19. Diabetes Metab Syndr. 2020 May-June; 14(3): 247-250. Published online 2020 Mar 25. doi: 10.1016/j.dsx.2020.03.013

6. Feng G, Zheng KI, Yan Q-Q, Rios RS, Targher G, Byrne CD, et al. COVID-19 and Liver Dysfunction: Current Insights and Emergent Therapeutic Strategies. J Clin Transl Hepatol. 2020 Mar 28; 8(1): 18-24. Published online 2020 Mar 30. doi: 10.14218/JCTH.2020.00018

7. Wollenberg A, Flohr C, Simon D, Cork MJ, Thyssen JP, Bieber T, et al. European Task Force on Atopic Dermatitis (ETFAD) statement on severe acute respiratory syndrome coronavirus 2 (SARS-Cov-2)infection and atopic dermatitis. J Eur Acad Dermatol Venereol. 2020 Jun;34(6):e241-e242. doi: 10.1111/ jdv.16411 [Epub ahead of print]

8. Ronco, C, Reis, T. Kidney involvement in COVID-19 and rationale for extracorporeal therapies. Nat Rev Nephrol. 2020 Apr 9 : 1-3. doi: 10.1038/s41581-020-0284-7 [Epub ahead of print]

9. Usher K, Durkin J, Bhullar N. The COVID-19 pandemic and mental health impacts. Int J Ment Health Nurs. 2020 Jun;29(3): 315-318. doi: https://doi.org/10.1111/inm.12726

10. Jiménez-Pavón D, Carbonell-Baeza A, Lavie CJ. Physical exercise as therapy to fight against the mental and physical consequences of COVID-19 quarantine: Special focus in older people. Prog Cardiovasc Dis. 2020 May-June; 63(3): 386-388. Published online 2020 Mar 24. doi: 10.1016/j.pcad.2020.03.009

11. Misra DP, Agarwal V, Gasparyan AY, Zimba O. Rheumatologists' perspective on coronavirus disease 19 (COVID-19) and potential therapeutic targets. Clin Rheumatol. 2020 Apr 10:1-8. doi: 10.1007/ s10067-020-05073-9 [Epub ahead of print]

12. Puig-Domingo M, Marazuela M, Giustina A. COVID-19 and endocrine diseases. A statement from the European Society of Endocrinology. Endocrine. 2020 Apr 11 : 1-4. doi: 10.1007/s12020-020-022945 [Epub ahead of print]

13. Lippi G, Henry BM, Sanchis-Gomar F. Physical inactivity and cardiovascular disease at the time of coronavirus disease 2019 (COVID-19). Eur J Prev Cardiol. 2020 Jun;27(9):906-908. doi: $10.1177 / 2047487320916823$ 
14. Mao L, Jin H, Wang M, Chen S, He Q, Chang J, et al. Neurologic Manifestations of Hospitalized Patients With Coronavirus Disease 2019 in Wuhan, China. JAMA Neurol. 2020 Jun; 77(6): 1-9. Published online 2020 Apr 10. doi: 10.1001/jamaneurol.2020.1127

15. Brasil. Secretaria Nacional dos Direitos da Pessoa com Deficiência. Ministério da Mulher, Família e Direitos Humanos. Cartilha Pessoas com Deficiência e com Doenças Raras e o COVID-19. Brasília: Ministério da Mulher, Família e Direitos Humanos; 2020.

16. Vardavas CI, Nikitara K. COVID-19 and smoking: A systematic review of the evidence. TobInduc Dis. 2020;18:20. Published 2020 Mar 20. doi:10.18332/tid/119324.

17. Grupo de Interesse em Fisioterapia Cardiorrespiratória. Gestão dos sintomas respiratórios na pessoa com COVID-19 [Internet]. [São Domingos de Rana]: Associação Portuguesa de Fisioterapeutas; 2020. Available from: https://www.gifcr-apf.com/

18. Grupo de Interesse em Fisioterapia Cardiorrespiratória. Fisioterapia respiratória em pessoas com COVID-19 [Internet]. [São Domingos de Rana]: Associação Portuguesa de Fisioterapeutas; 2020 May 4. Available from: https://www.gifcr-apf.com/

19. Thomas P, Baldwin C, Bissett B, Boden I, Gosselink R, Granger CL, et al. Physiotherapy management for COVID-19 in the acute hospital setting: clinical practice recommendations. J Physiother. 2020 Apr;66(2):73-82. doi: 10.1016/j.jphys.2020.03.011.

20. Guan W-j, Ni Z-y, Hu Y, Liang W-h, Ou C-q, He J-x, et al. Clinical Characteristics of Coronavirus Disease 2019 in China. N Engl J Med. 2020 Feb 28:NEJMoa2002032. Published online 2020 Feb 28. doi: 10.1056/NEJMoa2002032

21. Merhy EE, Feuerwerker LCM, Santos MLM, Bertussi DC, Baduy RS. Basic Healthcare Network, field of forces and micropolitics: implications for health management and care. Saúde debate [Internet]. 2019 Dec;43(spe6):70-83. doi: 10.1590/0103-11042019s606.

22. Merhy EE, Feuerwerker LMC, Gomes PC. Da repetição à diferença: construindo sentidos com o outro no mundo do cuidado. In: Feuerwerker LCM, Bertussi DC, Merhy EE, et al, organizadores. Avaliação compartilhada de saúde. Surpreendendo o instituído nas redes. Vol. 2. Rio de Janeiro: Hexis; 2016. p. 25-34.

23. Heidari F, Karimi E, Firouzifar M, Khamushian P, Ansari R, Mohammadi Ardehali M, et al. Anosmia as a prominent symptom of COVID-19 infection. Rhinology. 2020 Jun 1;58(3):302-303. doi: 10.4193/ Rhin20.140.

24. Wong SH, Lui RN, Sung JJ. Covid-19 and the digestive system. J Gastroenterol Hepatol. 2020 May;35(5):744-748. doi: 10.1111/jgh.15047.

25. Giavanetti EA, Klava KR, Cosme MR, e Silva RF, Kanda SS. Avaliação Geral. In: Gobbi FCM, Cavalheiro LV. Fisioterapia hospitalar - Avaliação e planejamento do tratamento fisioterapêutico. São Paulo: Atheneu; 2009. p. 22 -29.

26. Pereira CAC. Espirometria [Diretriz 1 - Diretrizes para testes de função pulmonar]. J Pneumol, 2002 Out;28(Supl 3):s1-s82.

27. Gazzotti M, Nogueira PAMS. Avaliação respiratória. In: Lanza FC, Gazzotti MR, Palazzin A, organizadores. Fisioterapia em pediatria e neonatologia da UTI ao ambulatório. São Paulo: Gen/Roca, 2012. p. 58. 
28. Presto B, Presto LDN. Fisioterapia Respiratória: uma nova visão. 2a. ed. rev. e ampl. Rio de Janeiro: BP; 2005. p. 71.

29. Pasterkamp H, Brand PL, Everard M, Garcia-Marcos L, Melbye H, Priftis KN. Towards the standardisation of lung sound nomenclature. Eur Respir J. 2016 Mar;47(3):724-32. doi: 10.1183/13993003.01132-2015.

30. Ruppel GL. Manual of pulmonary function testing. $7^{\text {th }}$ ed. Saint Louis: Mosby; 1998. p. 144.

31. Brasil. Ministério da Saúde. Máscaras caseiras podem ajudar na prevenção contra o Coronavírus [Internet]. Brasília: Ministério da Saúde; 2020. Available from: https://www.saude.gov.br/noticias/ agencia-saude/46645-mascaras-caseiras-podem-ajudar-na-prevencao-contra-o-coronavirus

32. WHO/OMS. EPI-WIN: WHO information network for epidemics. Geneva: WHO; 2020 [updated 2020 Jun 08]. Available from: https://www.who.int/teams/risk-communication

33. Ferreira MJ,Irigoyen MC, Consolim-Colombo F, Saraiva JFK, De Angelis K. Physically active lifestyle as an approach to confronting COVID-19. Arq Bras Cardiol [Internet]. 2020 Apr;114(4):601-2. Epub Apr 09, 2020. https://doi.org/10.36660/abc.20200235.

34. Martijn A. Spruit MA, Holland AE, Singh SJ, Troosters T, Balbi B, Berney S, et al. Report of an AdHoc international Task Force to develop an expert-based opinion on early and short-term rehabilitative interventions (after the acute hospital setting) in COVID-19 survivors (version April 3, 2020) [Internet]. Lausanne: European Respiratory Society; 2020. Available from: https://ers.app.box.com/s/ npzkvigtl4w3pb0vbsth4y0fxe7ae9z9

35. WHO/OMS. Clinical management of severe acute respiratory infection (SARI) when COVID-19 disease is suspected: Interim guidance, 13 March 2020 [Internet]. Geneva: World Health Organization; 2020. Available from: https://www.who.int/docs/default-source/coronaviruse/clinical-managementof-novel-cov.pdf

36. PAHO. Rehabilitation considerations during the COVID-19 outbreak Coronavirus [Internet]. Washington: Pan American Health Organization; 2020 [2020 May 25]. Available from: https://iris. paho.org/handle/10665.2/52035.

Submissão em: 09/06/2020

Aceito em: 15/07/2020 